\title{
Unsupervised Reconstruction of a Patient-Specific Surface Model of a Proximal Femur from Calibrated Fluoroscopic Images
}

\author{
Guoyan Zheng, Xiao Dong, and Miguel A. Gonzalez Ballester \\ MEM Research Center - ISTB, University of Bern, Stauffacherstrasse 78, \\ CH-3014, Bern, Switzerland \\ guoyan.zheng@ieee.org
}

\begin{abstract}
In this paper, we present an unsupervised 2D/3D reconstruction scheme combining a parameterized multiple-component geometrical model and a point distribution model, and show its application to automatically reconstruct a surface model of a proximal femur from a limited number of calibrated fluoroscopic images with no user intervention at all. The parameterized multiple-component geometrical model is regarded as a simplified description capturing the geometrical features of a proximal femur. Its parameters are optimally and automatically estimated from the input images using a particle filter based inference method. The estimated geometrical parameters are then used to initialize a point distribution model based $2 \mathrm{D} / 3 \mathrm{D}$ reconstruction scheme for an accurate reconstruction of a surface model of the proximal femur. We designed and conducted in vitro and in vivo experiments to compare the present unsupervised reconstruction scheme to a supervised one. An average mean error of $1.2 \mathrm{~mm}$ was found when the supervised reconstruction scheme was used. It increased to $1.3 \mathrm{~mm}$ when the unsupervised one was used. However, the unsupervised reconstruction scheme has the advantage of elimination of user intervention, which holds the potential to facilitate the application of the $2 \mathrm{D} / 3 \mathrm{D}$ reconstruction in surgical navigation.
\end{abstract}

Keywords: proximal femur, fluoroscopy, surface reconstruction, particle filter, multiple-component geometrical model, point distribution model.

\section{Introduction}

A patient-specific surface model of a proximal femur plays an important role in planning and supporting various computer-assisted surgical procedures including total hip replacement, hip resurfacing, and proximal femur osteotomy. Accordingly, various reconstruction methods have been developed.

One of these methods is to extract a three-dimensional (3D) surface model from volume data pre-operatively acquired from Computed Tomography (CT) or Magnetic Resonance Imaging (MRI) and then intra-operatively to register the extracted surface model to the patient anatomy. However, the high logistic effort and cost, the extra radiation involved with the CT imaging, and the

N. Ayache, S. Ourselin, A. Maeder (Eds.): MICCAI 2007, Part I, LNCS 4791, pp. 834 841, 2007.
(C) Springer-Verlag Berlin Heidelberg 2007 
large quantity of data to be acquired and processed make them less functional. The alternative is to reconstruct a patient-specific surface model from a limited number of intra-operatively acquired two-dimensional (2D) fluoroscopic images using a statistical model.

Several research groups have explored the methods for reconstructing a patient specific model from a statistical model and a limited number of calibrated X-ray images 1] 2 3] 4. Except the method presented in Yao and Taylor 1, which depends on a deformable $2 \mathrm{D} / 3 \mathrm{D}$ registration between an appearance based statistical model and a limited number of X-ray images, all other methods have their reliance on a point distribution model (PDM) in common. The common disadvantage of all these PDM based reconstruction methods lies in the fact that they require either knowledge about anatomical landmarks [4, which are normally obtained by interactive reconstruction from the input images, or an interactive alignment of the model with the input images [2] 3]. Such a supervised initialization is not appreciated in a surgical navigation application, largely due to the strict sterilization requirement.

To eliminate the user intervention constraint, we propose in this paper an unsupervised $2 \mathrm{D} / 3 \mathrm{D}$ reconstruction scheme combining a parameterized multiplecomponent geometrical model and a point distribution model, and show its application to automatically reconstruct a surface model of the proximal femur with no user intervention at all. The parameterized multiple-component geometrical model is regarded as a simplified description capturing the geometrical features of a proximal femur. The constraints between different components are described by a causal Bayesian network. A particle filter based inference algorithm [5] is applied to automatically estimate their parameters from the input X-ray images. The estimated geometrical parameters of the proximal femur are then used to initialize a point distribution model based 2D/3D reconstruction scheme for an accurate reconstruction of a surface model of the proximal femur.

This paper is organized as follows. Section 2 briefly recalls the supervised 2D/3D reconstruction scheme. Section 3 describes the approach for unsupervised initialization. Section 4 reports the experimental results, followed by conclusions in Section 5.

\section{Supervised 2D/3D Reconstruction Scheme}

\subsection{Image Acquisition}

We use calibrated fluoroscopic images. Due to the limited imaging volume of a fluoroscope, we ask for four images for the proximal femur from different view direction, of which two images focus on the proximal femoral head and the other two focus on the femoral shaft. The calibrated fluoroscopic image set is represented by $\mathbf{I}$. Although all four images are used to estimate the parameters of the multiple-component geometrical model, only those two images that focus on the proximal femur are used for surface reconstruction. 


\subsection{Statistical Model of the Proximal Femur}

The PDM used in this paper was constructed from a training database consisted of proximal femoral surfaces from above the less trochanter. Let $\mathbf{x}_{i}, i=$ $0,1, \ldots, m-1$, be $m=30$ members of the aligned training surfaces. Each member is described by a vectors $\mathbf{x}_{i}$ with $N=4098$ vertices:

$$
\mathbf{x}_{i}=\left\{x_{0}, y_{0}, z_{0}, x_{1}, y_{1}, z_{1}, \ldots, x_{N-1}, y_{N-1}, z_{N-1}\right\}
$$

The PDM is obtained by applying principal component analysis.

$$
\begin{aligned}
& \mathbf{D}=\frac{1}{(m-1)} \cdot \sum_{i=0}^{m-1}\left(\mathbf{x}_{i}-\overline{\mathbf{x}}\right) \cdot\left(\mathbf{x}_{i}-\overline{\mathbf{x}}\right)^{T} \\
& \sigma_{0} \geq \sigma_{1} \geq \cdots \geq \sigma_{m_{1}-1}>0 ; m_{1} \leq m-1 \\
& \mathbf{D} \cdot \mathbf{p}_{i}=\sigma_{i}^{2} \cdot \mathbf{p}_{i} ; i=0, \cdots, m_{1}-1
\end{aligned}
$$

where $\overline{\mathbf{x}}$ and $\mathbf{D}$ are the mean vector and the covariance matrix, respectively. $\left\{\sigma_{i}^{2}\right\}$ are non-zero eigenvalues of the covariance matrix $\mathbf{D}$, and $\left\{\mathbf{p}_{i}\right\}$ are the corresponding eigenvectors. The sorted eigenvalues $\sigma_{i}^{2}$ and the corresponding eigenvectors $\mathbf{p}_{i}$ are the principal directions spanning a shape space with $\overline{\mathbf{x}}$ representing its origin.

Then, an instance $\mathcal{M}$ generated from the statistical model with parameter set $\mathbf{Q}=\left\{s, \alpha_{0}, \alpha_{1}, \cdots, \alpha_{m_{1}-1}\right\}$ can be described as:

$$
\mathcal{M}: \mathbf{x}(\mathbf{Q})=s \cdot\left(\overline{\mathbf{x}}+\sum_{i=0}^{m_{1}-1}\left(\alpha_{i} \cdot \mathbf{p}_{i}\right)\right)
$$

where $s$ is the scaling factor; $\left\{\alpha_{i}\right\}$ are the weights calculated by projecting vector $(\mathbf{x} / s-\overline{\mathbf{x}})$ into the shape space. The mean surface model $\overline{\mathbf{x}}$ is shown in Fig. 1, left.

\section{$2.32 \mathrm{D} / 3 \mathrm{D}$ Reconstruction Scheme}

Our 2D-3D reconstruction scheme is a further improvement of the approach we introduced in 4, which combines statistical instantiation and regularized shape deformation with an iterative image-to-model correspondence establishing algorithm. The image-to-model correspondence is established using a non-rigid $2 \mathrm{D}$ point matching process, which iteratively uses a symmetric injective nearestneighbor mapping operator and 2D thin-plate splines based deformation to find a fraction of best matched 2D point pairs between features detected from the fluoroscopic images and those extracted from the 3D model. The image contours of the proximal femur are extracted from the input images by a graphical model based Bayesian inference 6] whereas the apparent contours of the 3D model are extracted using an approach described in 77. The obtained 2D point pairs are then used to set up a set of 3D point pairs such that we turn a 2D-3D reconstruction problem to a $3 \mathrm{D}-3 \mathrm{D}$ one. The $3 \mathrm{D} / 3 \mathrm{D}$ reconstruction problem is then solved optimally in three sequential stages. For details, we refer to our previous works 4 and 6 . 

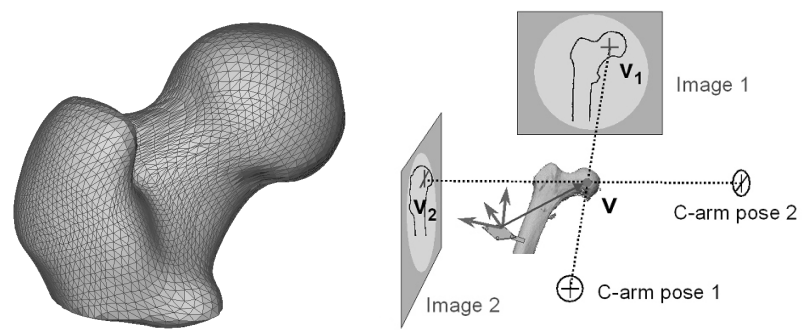

Fig. 1. The mean surface model of our point distribution model (left) and a schematic view of landmark reconstruction (right)

\subsection{Supervised Initialization}

The convergence of the $2 \mathrm{D} / 3 \mathrm{D}$ reconstruction scheme introduced in 4 relies on a proper initialization of scale and pose of the mean surface model of the PDM. In our previous work 4], three anatomical landmarks, i.e., the center of the femoral head, a point on the axis of the femoral neck, and the apex of the greater trochanter were reconstructed interactively from the input fluoroscopic images, as shown in Fig. 1, right, and were used to compute the initial scale $s_{0}$ and the initial rigid transformation $T_{0}$ of the mean surface model of the PDM in relative to the input images.

\section{Approach for Unsupervised Initialization}

\subsection{Proximal Femur Model}

The proximal femur is approximated by a simplified geometrical model consisting of 3 components: head, neck and shaft, which are described by a sphere, a trunked cone and a cylinder with parameter set $\mathbf{X}_{\text {Femur }}=\left\{\mathbf{X}_{\text {Head }}, \mathbf{X}_{\text {Neck }}, \mathbf{X}_{\text {Shaft }}\right\}$ respectively as shown in Fig. 2, left. These three components are constrained by the anatomical structure of the proximal femur. The advantage of using such a model is apparent. On the one hand, this simplified 3D model has the capability to catch the global structure of the anatomy from the fluoroscopic images and is not dependent on the view directions of the input images. On the other hand, using such a model to estimate the geometrical parameters of the proximal femur is much less computational expensive than using a point distribution model, largely due to the simple and parameterized geometrical shape of its components.

The constraints among components are represented by a causal Bayesian network as shown in Fig. 2, right, where all $\pi(\cdot)$ 's are prior distributions and all $p(\cdot)$ 's are conditional distributions. The prior distributions are designed according to the information estimated from the two images that focus on the proximal femur and the prior information about the geometrical features of each component, e.g., the centroids of three components are assumed uniformly distributed in the common view volume of the fluoroscopic images, which can be obtained by calculating the intersection of their projection frustums; the radii and the lengths (for 
neck and shaft) of different components are assumed to be uniformly distributed in their associated anatomical ranges. The structural constraints among components are set so that the component configuration that fulfills these constraints will show a higher probability of being assembled to represent a proper proximal femur. These constraints are regarded as the conditional distributions of those components when the configuration of their parent components is given. The reason why the network starts from shaft component is that the shaft component is much easier to be detected from the images than other two components, which will accelerate the convergence of the model fitting algorithm as described below.

\subsection{Geometrical Model Fitting by Particle Filter}

Particle filter, also known as the Condensation algorithm [8] is a robust filtering technique, based on the Bayesian framework. This technique provides a suitable basic framework for estimating paramerers of a multiple-component geometrical model from images: particle filter estimates the states by recursively updating sample approximations of posterior distribution. In this work, we implement a particle filter based inference algorithm as follows.

1. Initialization: Generate the first generation of particle set with $M$ particles $\left\{P_{i}^{0}=\mathbf{X}_{F e m u r, i}^{0}\right\}_{i=0, \ldots, M-1}$ from the proposal distributions

$$
\begin{aligned}
& q^{0}\left(\mathbf{X}_{\text {Shaft }}\right)=\pi\left(\mathbf{X}_{\text {Shaft }}\right) \\
& q^{0}\left(\mathbf{X}_{\text {Neck }}\right)=\pi\left(\mathbf{X}_{\text {Neck }}\right) q^{0}\left(\mathbf{X}_{\text {Shaft }}\right) p\left(\mathbf{X}_{\text {Neck }} \mid \mathbf{X}_{\text {Shaft }}\right) \\
& q^{0}\left(\mathbf{X}_{\text {Head }}\right)=\pi\left(\mathbf{X}_{\text {Head }}\right) q^{0}\left(\mathbf{X}_{\text {Neck }}\right) p\left(\mathbf{X}_{\text {Head }} \mid \mathbf{X}_{\text {Neck }}\right)
\end{aligned}
$$

2. Observation: Given the current generation of particle set, calculate the weight of each particle as $w_{i}^{n} \propto \operatorname{Prob}\left(\mathbf{I} \mid \mathbf{X}_{F e m u r, i}^{n}\right)$, where $\operatorname{Prob}\left(\mathbf{I} \mid \mathbf{X}_{\text {Femur, }}^{n}\right)$ is called observation model and is defined by the product of two items:

$$
p\left(\mathbf{I} \mid \mathbf{X}_{\text {Femur }, i}^{n}\right)=\prod_{(I \in \mathbf{I})} p_{E}\left(I \mid \mathbf{X}_{\text {Femur }, i}^{n}\right) \cdot p_{G}\left(I \mid \mathbf{X}_{\text {Femur }, i}^{n}\right)
$$

The first item $p_{E}\left(I \mid \mathbf{X}_{\text {Femur, } i}^{n}\right)$ measures discrepancies between extremal contours of the model obtained by simulating X-ray projection to the $I$ th image and the edges $E(I)$ extracted from the $I$ th image by applying a Canny edge detector. For details about this similarity measure, we refer to chapter 6 of 10 .

The second item $p_{G}\left(I \mid \mathbf{X}_{F e m u r, i}^{n}\right)$ measures differences between the intensity distribution of the projected silhouettes of the model and that of the fluoroscopic image along the profile normal to the projected extremal contours of the model. For details about this similarity measure, we refer to chapter 7 of [10].

3. Update: Update the proposal distributions as

$$
\begin{aligned}
& q^{n+1}\left(\mathbf{X}_{\text {Shaft }}\right)=\operatorname{NPDE}\left(w_{i}^{n}, \mathbf{X}_{\text {Shaft }, i}^{n}\right) \\
& q^{n+1}\left(\mathbf{X}_{\text {Neck }}\right)=\pi\left(\mathbf{X}_{\text {Neck }}\right) q^{n+1}\left(\mathbf{X}_{\text {Shaft }}\right) p\left(\mathbf{X}_{\text {Neck }} \mid \mathbf{X}_{\text {Shaft }}\right)
\end{aligned}
$$




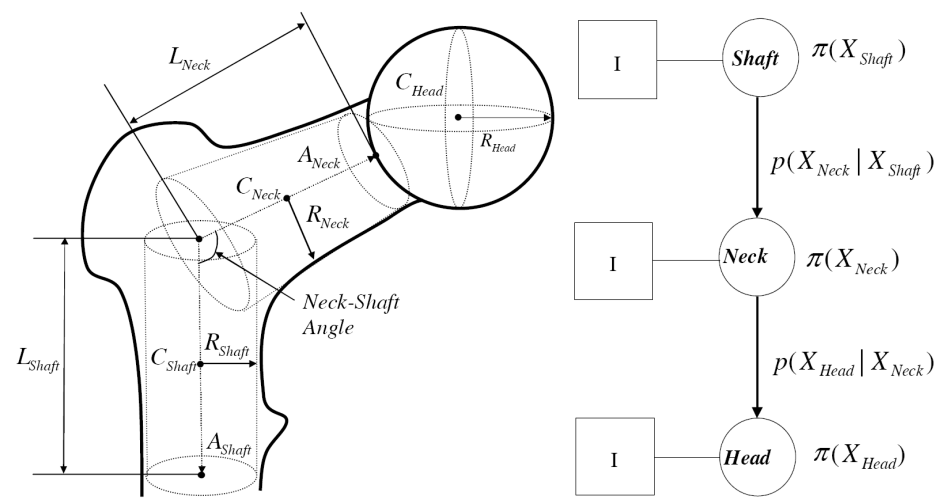

Fig. 2. The parameterized multiple-component geometrical model (left) and a causal Bayesian network for encoding the conditional distribution among components (right)

$$
q^{n+1}\left(\mathbf{X}_{\text {Head }}\right)=\pi\left(\mathbf{X}_{\text {Head }}\right) q^{n+1}\left(\mathbf{X}_{\text {Neck }}\right) p\left(\mathbf{X}_{\text {Head }} \mid \mathbf{X}_{\text {Neck }}\right)
$$

where $\operatorname{NPDE}\left(w_{i}^{n}, \mathbf{X}_{\text {Shaft,i }}^{n}\right)$ is a nonparametric density estimation [9] . Generate the next generation of particle set from the updated proposal distributions.

4. Go to 2 until the particle set converges.

\subsection{Unsupervised Initialization of the PDM}

From the mean surface model $\overline{\mathbf{x}}$ of the PDM, the model vertices can be classified into three regions, femoral head, neck and shaft. The femoral head center and radius, axes of femoral neck and shaft can be determined in the mean surface model coordinate space by a 3D sphere fitting to the femoral head region and cylinder fittings to the femoral neck and shaft regions. The initial rigid transformation and scale can then be computed to fit the PDM (the scaled mean surface model) to the estimated geometrical model of the proximal femur.

\section{Experimental Results}

We designed and conducted two experiments to validate the present approach. The first experiment was conducted on 3 clinical dataset. Due to the lack of ground truth, we used the clinical dataset to verify the robustness of the particle filter based inference algorithm. We run the algorithm for 10 trials on each dataset with particle number $M=200$. In each trial the proximal femur was correctly identified in about 4 minutes on a $3.0 \mathrm{GHz}$ Pentium IV computer with 1 GB RAM, when the algorithm was implemented with GCC 4.0 on a Fedora 4.0 Linux system. The statistical results are shown in Table 1. An example of the unsupervised initialization using the inference results is shown in Fig. 3

The second experiment was performed on 10 dry cadaveric femurs with different sizes and shapes. The purpose was to evaluate the accuracy of the unsupervised 2D/3D reconstruction. For each bone, two studies were performed. 


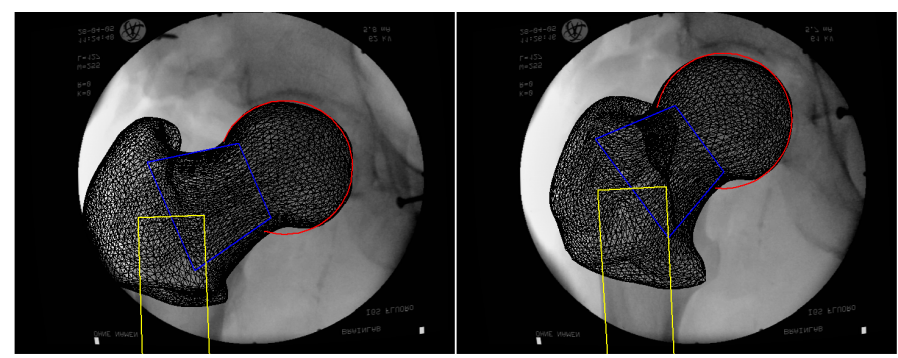

Fig. 3. An example of unsupervised initialization of the PDM. The color lines show the projected extremal contours of different components.

Table 1. Satistical results of the particel filter based inference algorithm, all results are relative to the mean values of the 10 trials

\begin{tabular}{l|c|c|c}
\hline Parameter & Data Set 1 & Data Set 2 & Data Set 3 \\
\hline Head Center $(\mathrm{mm})$ & $1.4 \pm 1.1$ & $0.1 \pm 0.1$ & $0.1 \pm 0.2$ \\
\hline Head Radius $(\mathrm{mm})$ & $0.3 \pm 0.4$ & $0.6 \pm 0.2$ & $1.0 \pm 0.8$ \\
\hline Neck Length $(\mathrm{mm})$ & $1.0 \pm 1.4$ & $1.3 \pm 1.8$ & $1.2 \pm 1.7$ \\
\hline Neck Axis (degree) & $0.8 \pm 0.7$ & $2.3 \pm 1.0$ & $1.8 \pm 1.1$ \\
\hline Shaft Radius $(\mathrm{mm})$ & $0.2 \pm 0.3$ & $0.1 \pm 0.2$ & $0.2 \pm 0.2$ \\
\hline Neck/Shaft Angle(degree) & $0.8 \pm 1.0$ & $2.0 \pm 2.5$ & $1.8 \pm 2.6$ \\
\hline
\end{tabular}

Table 2. The reconstruction errors when different initialization methods were used

\begin{tabular}{l|c|c|c|c|c|c|c|c|c|c|c}
\hline Bone Index & No. 1 & No. 2 & No. 3 & No. 4 & No. 5 & No. 6 & No. 7 & No. 8 & No. 9 & No. 10 \\
\hline Errors of supervised reconstruction \\
\hline Median $(\mathrm{mm})$ & 1.7 & 1.3 & 0.8 & 0.9 & 1.3 & 1.0 & 0.9 & 0.8 & 0.8 & 1.1 \\
\hline Mean $(\mathrm{mm})$ & 1.7 & 1.4 & 0.9 & 1.3 & 1.4 & 1.1 & 1.1 & 1.0 & 1.0 & 1.2 \\
\hline \multicolumn{10}{c}{ Errors of unsupervised reconstruction } \\
\hline Median $(\mathrm{mm})$ & 1.8 & 1.4 & 0.9 & 1.6 & 1.3 & 1.2 & 1.0 & 1.2 & 1.5 & 0.8 \\
\hline Mean $(\mathrm{mm})$ & 1.9 & 1.6 & 0.9 & 1.5 & 1.2 & 1.2 & 1.2 & 1.1 & 1.5 & 1.1 \\
\hline
\end{tabular}

In the first study, the $2 \mathrm{D} / 3 \mathrm{D}$ reconstruction scheme was initialized using the interactionvely reconstructed landmarks as described in Section 2, whereas in the second study, the present unspervised initialization was used to initialize the $2 \mathrm{D} / 3 \mathrm{D}$ reconstruction scheme. It took about 1 minute to interatctively reconstruct the landmarks for a supervised initilization for each case in the first study. To evaluate the reconstruction accuracy, 200 points were digitized from each bone surface. The distance between these points to the reconstructed surface of the associated bone were calculated and used to evaluate the reconstruction accuracy. The median and mean reconstruction errors for each study when using different initialization methods were recorded. The results are presented in Table 2. It was found that the unsupervised reconstruction was a little bit less accurate when compared to the supervised one. An average mean reconstruction error of 
$1.3 \mathrm{~mm}$ was found for the unsupervised reconstruction. It decreased to $1.2 \mathrm{~mm}$ when the supervised one was used.

\section{Conclusions}

In this paper, an unsupervised 2D/3D reconstruction scheme combining a parameterized multiple-component geometrical model with a point distribution model was presented. We solved the supervised initialization problem by using a particle filter based inference algorithm to automatically determine the geometrical parameters of a proximal femur from the calibrated fluoroscopic images. No user intervention is required any more. The qualitative and quantitative evaluation results on 3 clinical dataset and on dataset of 10 dry cadaveric bones indicate the validity of the present approach. Although the unsupervised reconstruction is a little bit less accurate and needs longer time than the supervised one, it has the advantage of elimination of user intervention, which holds the potential to facilitate the application of the $2 \mathrm{D} / 3 \mathrm{D}$ reconstruction in surgical navigation.

\section{References}

1. Yao, J., Taylor, R.H.: Assessing accuracy factors in deformable 2D/3D medical image registration using a statistical pelvis model. In: ICCV 2003, vol. 2 pp. 13291334 (2003)

2. Fleute, M., Lavallée, S.: Nonrigid 3D/2D registration of images using a statistical model. In: Taylor, C., Colchester, A. (eds.) MICCAI'99. LNCS, vol. 1679, pp. 138 147. Springer, Heidelberg (1999)

3. Benameur, S., Mignotte, M., Parent, S., et al.: A hierarchical statistical modeling approach for the unsupervised 3D biplanar reconstruction of the scoliotic spine. IEEE Trans. Biomed. Eng. 52, 2041-2057 (2005)

4. Zheng, G., Gonzalez Ballester, M.A., Styner, M., Nolte, L.-P.: Reconstruction of patient-specific 3D bone surface from $2 \mathrm{D}$ calibrated fluoroscopic images and point distribution model. In: Larsen, R., Nielsen, M., Sporring, J. (eds.) MICCAI 2006. LNCS, vol. 4190, pp. 25-32. Springer, Heidelberg (2006)

5. Dong, X., Zheng, G.: A computational framework for automatic determination of morphological parameters of proximal femur from intraoperative fluoroscopic images. In: ICPR 2006, Part I pp. 1008-1013 (2006)

6. Dong, X., Gonzalez Ballester, M.A., Zheng, G.: Automatic extraction of femur contours from calibrated fluoroscopic images. WACV 2007, p. 55 (2007)

7. Hertzmann, A., Zorin, D.: Illustrating smooth surface. In: SIGGRAPH 2000, pp. 517-526 (2000)

8. Isard, M., Blake, A.: Contour tracking by stochastic propagation of conditional density. In: Buxton, B.F., Cipolla, R. (eds.) ECCV 1996. LNCS, vol. 1064, pp. 343-356. Springer, Heidelberg (1996)

9. Scott, D.W.: Multivariate Density Estimation. Wiley, Chichester (1992)

10. Cootes, T., Taylor, C.: Statistical models of appearance for computer vision. Technical report, University of Manschester, United Kingdom, ch. 6 \& 7, pp. 34-42 (2004) 